\section{SP4-7 HEALTH STUDENTS' KNOWLEDGE AND NEEDS RELATING TO GLOBAL HEALTH AND HEALTH EQUITY: A PROVINCE- WIDE STUDY}

doi:10.1136/jech.2011.142976p.1

\begin{abstract}
${ }^{1,2} \mathrm{M}$ Veras, ${ }^{*}{ }^{1} \mathrm{P}$ Tugwell, ${ }^{1} \mathrm{~K}$ Pottie, ${ }^{1} \mathrm{~V}$ Welch. ${ }^{1}$ University of Ottawa, Ottawa, Ontario, Canada; ${ }^{2}$ Canadian Institutes of Health Research, Ottawa, Ontario, Canada
\end{abstract}

The purpose of this study is to evaluate the extent of global health content in the current curricula of family medicine, nursing and physiotherapy programs and to conduct a survey for students in order to evaluate the knowledge, attitudes and education needs about global health.

Methodology The global health content of medical, nursing and physiotherapy curricula will be assessed through a document analysis. The appraisal will include the printed and electronic documents of these programs from each of the six Ontario universities in Canada. Additionally, a survey will be validated and administrated to health students. The validation will cover face and content validity, reliability and internal consistence reliability. The sample size will be calculated with the Bland formula. Cronbach's $\alpha$ will assess the internal consistency of the instrument for a complete multi-question scale.

Results The preliminary results of the document analysis revealed a lack of global health content in the curricula of these programs. There was a lack of printed and electronic information about global health as well as a lack of information regarding the description of the global health programs, evaluation, mentorship, funding, objectives and challenges. We are currently in the process of collecting data for the quantitative study.

Conclusion The preliminary conclusion of this study showed that there is a lack of global health content in the nursing, family physician residency and physiotherapy programs. Additionally, there is a need to develop global health competence across disciplines in order to improve health outcomes for diverse and disadvantaged population and promote health equity.

\section{SP4-8 HEALTH HAZARD DUE TO EXPOSURE OF PESTICIDE IN DABALIAPARA AREA OF BARPETA DISTRICT, ASSAM, INDIA}

doi:10.1136/jech.2011.142976p.2

R Hazarika, ${ }^{*}$ J Das, S Das, K Misra, L Dey. M C College, Barpeta, Barpeta, Assam, India

Introduction Health hazards due to extensive use of pesticides (organochlorine (OC) and organophosphate (OP)) is obvious in the third world countries. Therefore to ensure the safety of workers in the field, health surveillance is required. OC and OP pesticides used in the field mainly consist of aldine, BHC, dimethe, phosphomedon, endosulfan, melathion, carbofluron.

Methods and Materials The present study was undertaken among the field workers in the Katazar area of Barpeta District who were employed in the handling of pesticides. Exposed agricultural workers were studied alongside100 control workers who were not spraying pesticides. Age, Sex and Blood pressure were measured in both group. Toxic symptoms were recorded including neurological and gastrointestinal disease.

Results Most of the farmers suffered from excessive sweating (41.3\%, $R R=1.65)$, burning of the eyes $(41.3 \%, R R=1.65)$, excessive salivation $(10.0 \%, R R=1.66)$, fatigue $(18 \%, R R=1.86)$, dizziness (25.5\%, $R R=1.45)$, muscle weakness $(9.3 \%, R R=1.12)$, blurred vision $(11.3 \%, R R=0.96)$, and chest pain $(9.3 \%, R R=0.97)$.

Conclusion The application of safety measures, public awareness and training of the farmers about the safe handling of pesticide along with the use of protective devices are suggested. In addition, the possible use of comparatively less toxic pesticides specifically in place of melathion, phosphomedon and BHC are suggested. The use of carcinoogenic pesticides and cancer rates among the workers in area is a further concern and area for future study.

\section{SP4-9 METABOLIC PROFILE INFLUENCE ON HBA1C IN DIAGNOSING DIABETES MELLITUS}

doi:10.1136/jech.2011.142976p.3

T Dzebisashvili.* Moscow Regional Research Clinical Institute named by M.F.Vladimirskiy, Moscow, Russia

Aims To evaluate the optimum HbA1c cut-off for lowing number of people with undiagnosed type 2 diabetes mellitus (T2DM).

Materials and Methods Population-based screening for glucose metabolism impairments (GMI) among 661 adults in Moscow Country was conducted in 2009. HbA1c was determined in 39 subjects with GMI. T2DM was diagnosed according to WHO 1999 criteria. Receiver operating characteristics (ROC) analysis was performed to assess best predictive cut-off $\mathrm{HbA1c}$ for diagnosing T2DM.

Results Based on OGTT and HbA1c, $15 \%$ and $28 \%$ people had T2DM. Area under ROC curve (AUROC) was 0.727 (95\% CI 0.490 to $0.964, p=0.080$, sensitivity $66.7 \%$, specificity $78.8 \%$ ) using $\mathrm{ADA}$ recommended $\mathrm{HbA} 1 \mathrm{c}$ cut-off $>6.5 \%$. Best predictive $\mathrm{HbA1c}$ in this cohort was $6.3 \%$ (AUROC $0.750, p=0.054$, sensitivity $83 \%$, specificity $67 \%$ ). $33.0 \%$ of undiagnosed T2DM had HbA1c levels $<6.5 \%$ ( $95 \%$ CI $0 \%$ to $71 \%$ ) and $17 \%$ of people with T2DM had HbA1c levels $<6.3 \%$. Subjects with false negative HbA1c were predominantly with normal BMI $(21.8+1.6$ vs $42.9+7.8, p=0.025)$, false positives were predominantly with higher BMI $(30.6+7.8$ vs $28.4+$ $5.9, \mathrm{p}=0.273$ ). In normal weight (BMI 18-25) individuals optimal HbA1c cut-point for detecting T2DM was $>6.0 \%$ (AUROC 0.750 , sensitivity $50 \%$, specificity $100 \%)$. RR of T2DM was 7 (1.18-42.9) with HbA1c values $6.0-6.4 \%$, than those with $<6.0$ in normal weight individuals.

Conclusion Choosing the HbA1c strategy rather than the OGTT strategy leads to diagnose more diabetes, although the consistency of both diagnostic criteria is low. The optimal HbA1c cut-point to detect T2DM was lower than $\mathrm{HbA1c}$ of $6.5 \%$ in normal weight individuals.

\section{SP4-10 PREVALENCE AND FACTORS ASSOCIATED WITH IRRITABLE BOWEL SYNDROME AMONG MEDICAL STUDENTS OF KARACHI: A CROSS-SECTIONAL STUDY}

doi:10.1136/jech.2011.142976p.4

S S Naeem, ${ }^{*}$ E U Siddiqui. Dow University of Health Sciences, Karachi, Sind, Pakistan

Introduction Irritable bowel syndrome is commonly reported among university students, however few analytical based studies are available on IBS from Pakistan. We investigate the prevalence and pattern of symptoms of IBS along with anxiety among medical students of Karachi.

Methods A cross-sectional study was conducted among 360 medical students of Karachi. Data were collected using validated tool "Rome III Criteria" and Generalised Anxiety Disorder Questionnaire. Diagnosis were made on the criteria that students experiencing abdominal discomfort at least 2-3 days/month, also had high level of Anxiety. Convenient sampling was done to recruit the participants after getting written informed consent.

Results The prevalence of IBS was found to be 102 (28.3\%) with a predominance of 87 (85.29\%) in females than males $15(14.71 \%)$. 